E3S Web of Conferences 1, 14002 (2013)

DOI: $10.1051 / \mathrm{e} 3$ sconf/20130114002

(c) Owned by the authors, published by EDP Sciences, 2013

\title{
Total level and release of silver from a nanoparticles containing dressing used in burns care - a pilot study
}

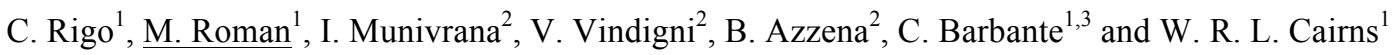 \\ ${ }^{1}$ Institute for the Dynamics of Environmental Processes (IDPA-CNR), Dorsoduro 2137, 30121 Venice, Italy \\ ${ }^{2}$ Clinics of Plastic and Reconstructive Surgery and Burn Centre, Padua University School of Medicine, Via Giustiniani \\ 2, 35128 Padova, Italy \\ ${ }^{3}$ University Ca' Foscari of Venice, department of Environmental Sciences, Dorsoduro 2137, 30121 Venice, Italy
}

\begin{abstract}
For centuries silver and its compounds have been in use to control infection and avoid septicaemia in the care of burns and chronic wounds. Renewed interest has resulted in a number of silver-based dressings exploiting nanotechnology that are now widely employed in burns centers. Despite extensive use, a systematic study of the chemical composition, release kinetics and biochemical action of these products has yet to be published. In this work we have characterized the morphology and elemental composition of a commercial dressings containing Ag by SEM-EDS. The silver content was determined by ICP-MS to be about $1.4 \mathrm{mg} \mathrm{cm}^{-2}$. Release kinetics in ultra pure water, physiological saline solution and human serum substitute were then deeply investigated. The highest release rates were found in serum substitute, with a maximum of $2.6 \mu \mathrm{g} \mathrm{hr}^{-1} \mathrm{~cm}^{-2}$. Our results show that the mean inhibitory concentrations are exceeded for most common pathogens in serum substitute and sterile water, while the presence of high $\mathrm{Cl}^{-}$ levels inactivates the dressings.
\end{abstract}

Key words: Silver, Burns, ICP-MS, isotope dilution analysis, SEM-E

\section{Introduction}

For centuries silver (Ag) has been used for its antimicrobial properties to control infection and avoid septicaemia in the care of burns and chronic wounds. Due to the local interruption of blood flow in burns, systemic infection prophylaxes have little effect, so topical antimicrobial treatments become decisive. In the 17th century, silver nitrate became the first Ag salt to be systematically applied in the treatment of chronic wounds and ulcers. However it was only after the publication by Moyer et al., 1965 that use of $\mathrm{Ag}$ diversified into a variety of products marketed for the treatment of burns. These products include colloidal Ag, chloride and sulphate solutions, creams and a number of dressings impregnated with Ag.

The recent discovery that nano-sized particles (NPs) display extremely innovative properties provided an immense momentum to the development of new consumer and medical products based on nano-Ag technology. Products containing silver NPs comprised $30 \%$ of the nanotechnology industry in 2009 and are indicated nowadays as one of the fastest growing field (Schafer et al., 2011). Still, the antimicrobial activity is the major direction for development of nano-Ag products.

While these products are already in use, still there is no specific European, American or international standard on the toxicology and biocompatibility of Ag NPs. Even if for some products toxicity testing, at least to some extent, is required prior to its placing on the market, for most consumer products limited or even no information on the risks of nano-Ag uses is freely accessible. Comprehensive crucial information is lacking on the concentrations of $\mathrm{Ag}$ in the products, the size and the form in which it is present (aggregates, agglomerates), the putative exposure routes and the probability of release (migration/abrasion) from the materials (Poda et al., 2011). Also, the real toxicokinetics, biochemical pathways and impacts of nano-Ag in the human body are still largely unknown because there is no standardization for Ag antimicrobial testing methods, toxicological data for patients are mostly unavailable and the complex analytical issues affect the determination of silver and its species.

Our research group recently launched a project aimed to the study of some Ag-based dressings among the most widely used. Our primary objective is to elucidate the chemistry and toxicology of Ag dressings in the treatment of burns and chronic skin wounds. This 
implies answering the following key questions: 1) what is the chemical behaviour of $\mathrm{Ag}$ applied to the human skin? 2) Are Ag containing products toxic for the patients? 3) How chemical and toxicological information can be combined to improve the design and use of these products? Here we present the first phase of a pilot work we carried out to determine the total Ag level in a dressing among those are daily used in the Burns Center at the University Hospital of Padova (Italy). The dressings was also characterized by SEM-EDS analysis and the release kinetics of Ag was studied in vitro.

\section{Materials and Methods}

The investigated dressing consist of a flexible polyethylene cloth coated with nanocrystalline Ag.

A wide range of tests aimed to the complete mineralization of the polymeric material were carried out by dissolution in organic solvents, acid digestion in a hot block with COD tubes, muffle furnace dry ashing followed by hot plate acid digestion and microwave oven acid digestion. Two specifically optimized methods were finally selected. Total Ag concentration was determined in the digests by ICP-QMS model 7500 from Agilent Technologies (Tokyo, Japan). Since no certified reference materials are available for the same matrix/Ag level of our interest, the recovery of digestions was assessed by standard additions and validated by applying both external calibration and isotope dilution analysis (IDA) for the quantification of $\mathrm{Ag}$ in the same samples.

The dressing was then used to perform in vitro $\mathrm{Ag}$ release experiments. The release was assessed in three matrices of increasing complexity: ultra pure water, physiological saline solution and human serum substitute (containing human albumin, insulin and transferrin in a buffered media at the typical concentrations observed in real human serum), in static conditions at $37^{\circ} \mathrm{C}$. Aliquots of the solutions were sampled at selected time steps up to the maximum use time suggested by the producer.

\section{Results and Discussion}

A basic approach was finally selected to reach the complete mineralization of polyethylene consisting of dry ashing in muffle furnace and then acid digestion of the residue on a hot plate. An alternative method based on microwave acid digestion was also optimized following a specific temperature program. Both methods were assessed in terms of $\mathrm{Ag}$ recovery obtaining $\sim 80 \%$ for the basic method and quantitative recovery for microwave digestion. However, accuracy was not statistically different thus we hypothesized that during dry ashing the spiked standard got partially lost while Ag from the sample did not. Each determination was carried out by external calibration and validated by IDA. No significant differences were revealed between the two approaches in terms of accuracy, but the latter showed significantly higher reproducibility as assessed by the intra-sample correlation (ICC, $>0.90$ for IDA and $<0.60$ for external calibration). Our final results confirmed the Ag levels declared by producer, corresponding to $\sim 120$ $\mathrm{mg} / \mathrm{g}$ with low variability within and between units of the same material. The total Ag concentration in this dressing is one of the highest among the products currently marketed for the care of burns.

The SEM analysis (Figure 1) confirmed that each fibre is homogeneously coated with a layer of aggregated Ag nanoparticles whose size ranges between $200 \mathrm{~nm}$ and 450 $\mathrm{nm}$.

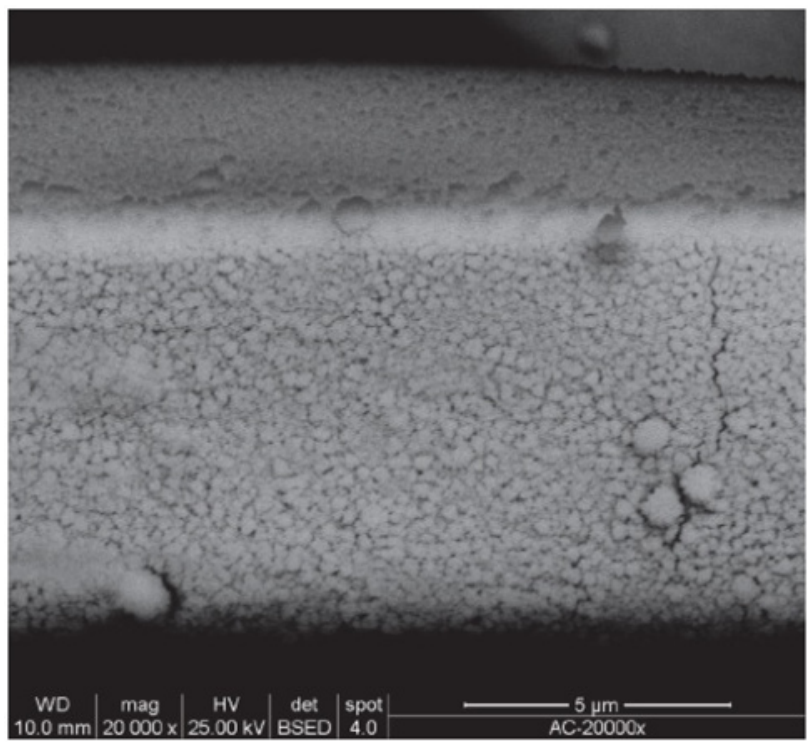

Fig. 1. SEM image of a single polyethylene fibre coated with agglomerates of $\mathrm{Ag}$ nanoparticles.

The dressing was then used to investigate the kinetics of Ag release in three different matrices of increasing complexity. Figure 2 represents the curves of $\mathrm{Ag}$ concentration in solutions as a function of the incubation time. The release curves show that although the producer suggests a maximum application time of 3 days, the dressing continues to release $\mathrm{Ag}$ up to this time and probably beyond, even if at a reduced rate. After 3 days the Ag released even into serum substitute (the highest value obtained) was only $7 \%$ of the total level in the original dressing. The release rate into saline solution appears to be very low, due to the formation of insoluble $\mathrm{AgCl}$ on the surface of the fibres.

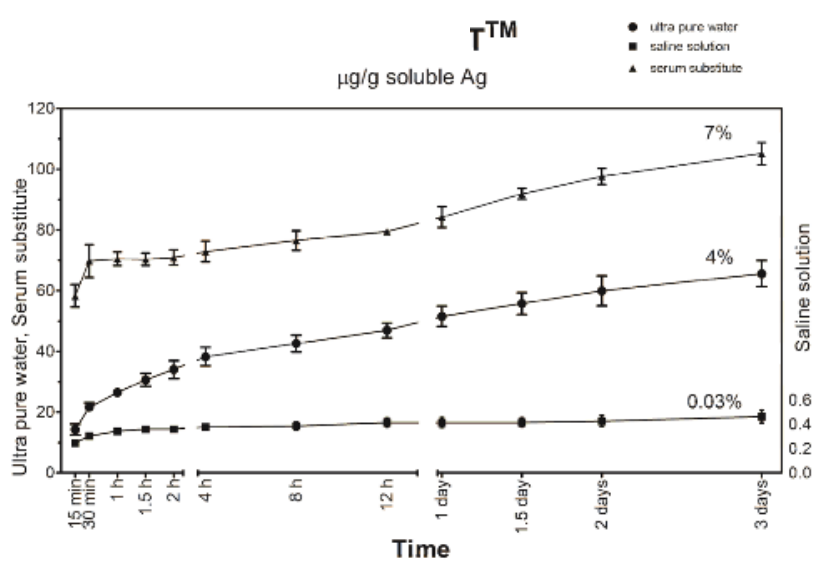

Fig. 2. Soluble Ag concentration released by the product in ultrapure water, physiological saline solution and human serum substitute. 
The deposited $\mathrm{AgCl}$ crystals form a barrier that may prevent the formation and transfer of other $\mathrm{Ag}^{+}$ions into solution, effectively deactivating the dressing ( $\mathrm{Li}$ et al., 2011). Since skin wounds and burns are $\mathrm{Cl}^{-}$rich environments we discourage the practice of moistening this product using physiological saline solution instead of sterile water to increase bioavailability of Ag. The highest release rate was observed in human serum substitute probably due to complexation with proteins, particularly albumin (Clem Gruen, 1975). This hypothesis was confirmed by size-exclusion (SEC) high performance liquid chromatography (HPLC) coupled on-line with diode-array (DAD-UV) and ICP-MS detectors, whose chromatograms confirmed that $\mathrm{Ag}$ in mainly bonded to the albumin fraction.

When considering some of the most common pathogens recovered from burn patients (i.e. Pseudomonas aeruginosa, Acinetobacter spp, Klebsiella spp, Staphylococcus aureus MRSA, E. Faecalis, E. Faecium, E. Coli and Candida spp) our results show that after one day in serum substitute the dressing releases the minimal inhibitory concentration of the soluble Ag necessary to reduce their replication. In normal saline solution at the end of the maximum application time the soluble $\mathrm{Ag}$ concentration is not enough to inhibit the growth of any of the microbes mentioned above. These results support other studies suggesting that moistening the dressing with normal saline solution should be avoided to maintain the activity of $\mathrm{Ag}$ and its antimicrobial properties (Asz et al., 2006).

\section{Conclusion}

The results of release experiments carried out in this research work attest that the highest release rate is found in serum substitute and the lowest release rate is in saline solution. The low apparent release rates in saline solution are due to the formation of insoluble $\mathrm{AgCl}$. Re-solubilization and analysis of the precipitate demonstrates that higher amounts of $\mathrm{Ag}$ were released but precipitated immediately rendering the released $\mathrm{Ag}$ inactive. We believe that the $\mathrm{Ag}$ released into serum substitute is largely bound to the proteins, so it remains soluble even in the presence of high $\mathrm{Cl}^{-}$levels. This binding is expected to shift the release equilibrium by removing ionic $\mathrm{Ag}$ from solution resulting in faster release of the metal from the dressing's surface. Finally, despite that the release experiments were carried out for the maximum application time reported on the information leaflet of the dressing (3 days), when only minimal fractions of Ag had been released by the product Work is in progress to integrate our in vitro evidences with observation of real patients. Due to the rapidly increasing use of medical products based on nano-Ag technology, we hope that in the near future further scientific investigations will be carried out to prolong Ag release from these dressings and to reduce the amount of $\mathrm{Ag}$ thrown away or discharged into environment.

\section{Acknowledgements}

The authors thank the financial supports providing from Italian MIUR-FIRB funding (Project RBFR08M6W8).

\section{References}

Asz J, Asz D, Moushey R, Seigel J, Mallory SB, Foglia RP. Treatment of toxic epidermal necrolysis in a pediatric patient with a nanocrystalline silver dressing. J Pediatr Surg 2006; 41: e9-e12.

Clem Gruen L. Interaction of amino acids with silver(I) ions. BBA-Proteins Struct M 1975; 386: 270-274.

Li X, Lenhart JJ, Walker HW. Aggregation Kinetics and Dissolution of Coated Silver Nanoparticles. Langmuir 2011; 28: 1095-1104.

Moyer CA, Brentano L, Gravens DL, Margraf HW, Monafo W, JR. Treatment of Large Human Burns With $0.5 \%$ Silver Nitrate Solution. AMA Arch Surg 1965; 90: 812-867.

Poda AR, Bednar AJ, Kennedy AJ, Harmon A, Hull M, Mitrano DM, Ranville JF, Steevens J. Characterization of silver nanoparticles using flow-field flow fractionation interfaced to inductively coupled plasma mass spectrometry. J Chromatogr 2011; 1218: 4219-4225.

Schafer B, Tentschert J, Luch A. Nanosilver in Consumer Products and Human Health: More Information Required! Environ Sci Technol 2011; 45: 7589-7590. 